\title{
Electron impact dissociative excitation of water within the adiabatic nuclei approximation
}

\author{
J. D. Gorfinkiel, L. A. Morgan and Jonathan Tennyson \\ Department of Physics and Astronomy, University College London, Gower Street, \\ London WC1E 6BT, UK
}

\begin{abstract}
.
The $R$-matrix method is used to calculate dissociative excitation cross sections for the four lowest-lying electronically excited states of $\mathrm{H}_{2} \mathrm{O}$ in the energy range $5 \mathrm{eV}$ $15 \mathrm{eV}$. For the first time calculations are performed taking into account the nuclear motion by means of an adaptation of the adiabatic nuclei approximation. Cross sections are compared with previous and new fixed-nuclei results and also experiments. Resonance positions and widths are calculated for different geometries of the water molecule.
\end{abstract}

PACS numbers:

\section{Introduction}

Water is an extremely important molecule: it is the main constituent of living organisms; it is present in the atmosphere, space environments and is a solvent in many chemical processes. The damage caused by ionizing radiation on biological matter, in particular on DNA, has been an important field of experimental research in the last few years (see Boudaiffa et al (2000) and references therein). Structural water molecules are always present in living matter and the (secondary) electrons responsible for radiation damage have energies between 1 and $20 \mathrm{eV}$.

Experimental work on electron water collisions has been mainly restricted to dissociative attachment (for example, Belic̀ et al 1981, Curtis and Walker 1992, Melton 1972), photodissociation (e.g. Mordaunt et al 1994, Hwang et al 1999) and energy-loss spectra (Lassetre et al 1968, Trajmar et al 1973). Photodissociation has also been extensively studied theoretically (e.g. Engel et al 1992, Yang et al 2000, Schinke 1993, van Harrevelt and van Hemert 2000). Fragmentation of water in gas phase has been studied experimentally in various ways (e.g. Tsurubichi et al 1974, Kurawaki et al 1983) but only recently have measurements of the dissociation of water specifically via electron impact excitation been made available (Harb et al 2001). Fragmentation of water in solid amorphous state has also been studied (Rowntree et al 1991, Kimmel and Orlando 1995). Few theoretical calculations on dissociative 
excitation of water are available (Morgan 1998, Gil et al 1994, Lee et al 1993, Pritchard et al 1990) and all these studies neglect nuclear motion.

In this work, we calculate total (integral) cross sections for excitation into the four lowest states of water. We use the $R$-matrix method and the $R$-matrix polyatomic code (Morgan et al 1997) to deal with the electronic part of the process. To take into account the nuclear motion, we assume that dissociation will proceed into the formation of $\mathrm{H}$ and $\mathrm{OH}$ and use the energy balancing method (Stibbe and Tennyson 1998a, Trevisan and Tennyson 2001). This method is an adaptation of the adiabatic nuclei approximation that allows for the features of the dissociation process to be taken into account.

The ionization potential of water is $12.62 \mathrm{eV}$ (NIST 2001): for energies of the incoming electron higher than this value, this process will start to have some importance and so will excitation to other electronic states of water. For this reason, we limit our calculation to $15 \mathrm{eV}$. For the purpose of comparison, we also calculate cross sections using the fixed-nuclei approximation. Resonances positions and widths are determined for a set of molecular geometries.

All magnitudes are given in atomic units, unless otherwise stated.

\section{Method}

The basic idea of the $R$-matrix method is the division of configuration space into two regions. The boundary between the regions is defined by a sphere of a given radius $a$ centred at the centre of mass of the molecule. In the inner region, exchange and correlation are taken into account using rigorous quantum chemistry methods. In the outer region, where these effects are negligible, the use of a long-range multipole potential suffices to describe the electron-molecule interaction. The application of the $R$-matrix method to polyatomic molecules within the fixed-nuclei approximation has been described in detail elsewhere (Morgan et al 1997, Morgan et al 1998).

Previous calculations on electron-water scattering were carried out using the fixednuclei approach. This means that nuclear motion was neglected and calculations performed at the equilibrium geometry of the molecule. In order to include the nuclear motion we use the energy balancing method (see Stibbe and Tennyson 1998a, Trevisan and Tennyson 2001). This method is an extension of the adiabatic nuclei approximation (Chase 1956, Hara 1956, Shugard and Hazi 1975) that takes into account that the excess energy of the incoming electron can be split in any proportion between the dissociating fragments and the outgoing electron. It also allows for the inclusion of tunnelling effects.

In this work, we treat the nuclear dynamics of water as a pseudo-diatomic molecule. In its equilibrium geometry, the length of both $\mathrm{O}-\mathrm{H}$ bonds is $r_{O H}=1.81$ and the angle between them is $\alpha=104.5^{\circ}$. The ground state equilibrium bond length of $\mathrm{OH}$ is 1.83 (NIST 2001). Since this two values are very similar, it is possible to assume that dissociation into $\mathrm{H}$ and $\mathrm{OH}$ will take place via stretching of a single $\mathrm{OH}$ bond. Consequently, in our calculations, we fix one of the $\mathrm{O}-\mathrm{H}$ bonds and the angle at its equilibrium values and change the other bond length in the range $[1.3 ; 2.6]$. 
In the energy balancing method, off-shell fixed-nuclei T-matrices are calculated for a range of $\mathrm{OH}$ distances and then averaged using the initial (bound), $\Xi_{i \nu}\left(r_{O H}\right)$, and final (continuum), $\Xi_{j c}\left(E_{k e}, r_{O H}\right)$, nuclear wavefunctions:

$$
T_{i \nu \rightarrow j c}\left(E_{\text {in }}, E_{\text {out }}\right)=\left\langle\Xi_{j c}\left(E_{k e}, r_{O H}\right)\left|T_{i j}\left(\epsilon\left(r_{O H}\right)+E_{\text {out }}, E_{\text {out }}, r_{O H}\right)\right| \Xi_{i \nu}\left(r_{O H}\right)\right\rangle(1)
$$

where $i$ and $j$ denote the initial and final electronic states, $\nu$ denotes the initial vibrational state, $E_{k e}$ and $E_{\text {out }}$ are, respectively, the relative kinetic energy of the dissociating fragments and the energy of the scattered electron and $\epsilon_{j}\left(r_{O H}\right)$ corresponds to the energy threshold for excitation into the state $j$ for each value of $r_{O H}$. The $\mathrm{T}_{i j}$ are purely electronic and are calculated using the standard $R$-matrix procedure. The total cross section for excitation into each electronic dissociative state is calculated by integrating over all possible energies of the outgoing electron the energy-differential cross sections (in atomic units):

$$
\frac{\mathrm{d} \sigma_{i \nu \rightarrow j}}{\mathrm{~d} E_{\text {out }}}=\frac{\mu}{4 \pi^{3}} \frac{E_{k e}}{E_{\text {in }}} \sum_{S \Gamma l_{i} l_{j}}(2 S+1)\left|T_{i \nu l_{i} j c l_{j}}^{S \Gamma}\left(E_{\text {in }}, E_{\text {out }}\right)\right|^{2}
$$

where $\mu$ is the reduced mass of the system, $S$ is its total spin, $\Gamma$ defines its spatial symmetry, $E_{i n}$ is the energy of the incoming electron and $l_{i}$ and $l_{j}$ are the partial waves associated with the incoming and outgoing channels respectively. The explicit relation between the $\mathrm{T}_{i \nu l_{i j c l}}^{S \Gamma}$ and $\mathrm{T}_{i \nu \rightarrow j c}$ can be found in Trevisan and Tennyson (2001).

The electronic wavefunctions of the system are calculated using standard quantum chemistry methods (see section 3.1). The nuclear ground and continuum vibrational wavefunctions are calculated using the program LEVEL (Le Roy 1996) and directly solving the Schrödinger equation, respectively. The potentials required (that of the ground electronic state of water and those of the different electronic excited states) are explicitly calculated in the range $r_{O H} \in[1.0 ; 2.6]$ and then extrapolated to 8.0. The ones corresponding to singlet states are extrapolated using the data of van Harrevelt and van Hemert (2000) and the triplet state potentials using analytical exponential functions. As a test we extended the extrapolation range to 18.0: no significant change in the shape of the wavefunctions was observed.

\section{Characteristics of the calculation}

\subsection{Target states}

In order to obtain the target electronic wavefunctions, we first perform a Hartree-Fock self-consistent-field (HF-SCF) calculation using the double-zeta plus polarization (DZP) gaussian basis set of Dunning (1970) for O and the triple-zeta (TZ) basis of Dunning (1971) for $\mathrm{H}$ augmented with a diffuse s function and two $\mathrm{p}$ functions extracted from the basis set used by Gil et al (1994).

Using the molecular orbitals thus obtained, pseudo-natural orbitals (NOs) for all the targets states included in the calculation are generated from configuration interaction (CI) calculations of the order of 35000 and 55000 configurations for the singlets and 
triplets respectively. To obtain a set of NOs that is good for the representation of all the target states in the whole $r_{O H}$ range, we perform a weighted averaging of them. After several tests, we found that the best choice (i.e., the one that provides the best set of threshold energies and dipole moment for the ground state) is one that includes NOs from the ground state ${ }^{1} \mathrm{~A}_{1}(\tilde{X})$, the $1^{3} \mathrm{~B}_{1}$ and the ${ }^{1} \mathrm{~B}_{1}(\tilde{A})$ with weights 4,3 and 2 respectively. The same weights are used for all molecular geometries where these states are labelled as ${ }^{1} \mathrm{~A}^{\prime}(\tilde{X}), 1^{3} \mathrm{~A}^{\prime \prime}$ and ${ }^{1} \mathrm{~A}^{\prime \prime}(\tilde{A})$ respectively $\ddagger$. Finally, a CASCI (complete active space configuration interaction: all the possible configurations arising from distributing the electrons among the orbitals in the active space are built) calculation is performed. In our model, the $1 \mathrm{a}_{1}$ orbital was doubly occupied and all the excitations in the active space $\left(2 a_{1}, 3 a_{1}, 4 a_{1}, 5 a_{1}, 1 b_{1}, 2 b_{1}, 1 b_{2}\right)^{8}$ were taken into account. Some tests were performed in the direction of using more elaborate models for the target wave functions, in particular CASSD. In this model, configurations with single and double excitations from the active space into a limited space of target virtual orbitals are also included. We found that it was impossible to maintain the balance between the N-electron target representation and the $(\mathrm{N}+1)$-electron collision complex (for a discussion about this problem see Tennyson (1996)) and therefore opted for the CAS model.

The ground state energy for the equilibrium geometry is -76.09230 , which can be compared with the value of van Harrevelt and van Hemert (2000), -76.2905; the resulting dipole and quadrupole moments are $\mu=0.7334$ (the experimental value is $\mu=0.7295$ ), $Q_{0}=0.02656$ and $Q_{2}=-1.9715$. Energy differences between the ground and excited states, listed in table 1 , are in good agreement with previous calculations.

The same model is used to calculate the target properties for the non-equilibrium geometries (the active space is now $\left.\left(2 \mathrm{a}^{\prime}, 3 \mathrm{a}^{\prime}, 4 \mathrm{a}^{\prime}, 5 \mathrm{a}^{\prime}, 6 \mathrm{a}^{\prime}, 1 \mathrm{a}^{\prime \prime}, 2 \mathrm{a}^{\prime \prime}\right)^{8}\right)$. Fewer results are available for comparison. In the case of the singlet states, van Harrevelt and van Hemert (2000) performed MRDCI calculations for several states and several geometries. Our threshold energies are compared with their results in figure 1 . As can be seen, the agreement is good for the ground state and the 2 lowest singlet states. In the case of the ${ }^{1} \mathrm{~A}^{\prime \prime}$ and ${ }^{1} \mathrm{~A}^{\prime}\left(\tilde{E}^{\prime}\right)$ states our energies are too high over the whole range. These two states are known to be diffuse; in particular the $2^{1} \mathrm{~A}^{\prime \prime}$ state is dominantly of Rydberg character. Since our basis set (and therefore our natural orbitals) has to be contained inside the $R$ matrix sphere, diffuse states cannot be accurately represented. Nevertheless, we include these states in our calculation (as well as their corresponding triplets) as a way of accounting for the polarization of the target in the presence of the incoming electron.

To our knowledge, and somewhat surprisingly, there appears to be no previous data for the triplet state energies for non-equilibrium geometries.

$\ddagger$ The water molecule in its equilibrium geometry belongs to the $\mathrm{C}_{2 v}$ point group. The singlestretch geometries belong to the $\mathrm{C}_{s}$. Throughout the text, we will make reference to the irreducible representations from both point groups to label the states and orbitals. See table 1 for correspondence between the 2 sets of labels. 


\subsection{Scattering Model}

We perform the scattering calculations using two models as a way of testing the convergence of our results. Model (a) includes 7 target states: ${ }^{1} \mathrm{~A}^{\prime}(\tilde{X})$ (ground state), $1^{3} \mathrm{~A}^{\prime \prime},{ }^{1} \mathrm{~A}^{\prime \prime}(\tilde{A}), 1^{3} \mathrm{~A}^{\prime},{ }^{1} \mathrm{~A}^{\prime}(\tilde{B}), 2^{3} \mathrm{~A}^{\prime},{ }^{1} \mathrm{~A}^{\prime}\left(\tilde{E}^{\prime}\right)$. Model (b) includes 9 targets states: the ones from the previous model plus the $2^{3} \mathrm{~A}^{\prime \prime}$ and ${ }^{1} \mathrm{~A}^{\prime \prime}$. For model (a) an $R$-matrix radius of $a=10$ is sufficient, but inclusion of the diffuse $A^{\prime \prime}$ states means that, in order to make sure that all target wave functions are contained inside the inner region, $a=13$ is used for model (b).

The continuum orbitals describing the scattered electron are represented using gaussian type orbitals (GTOs) with $l \leq 4$ centred at the molecule centre of mass. The sets of optimized GTOs are obtained by fitting numerical Bessel functions over a finite range (Nestmann and Peyerimhoff 1990) that is usually slightly bigger than that of the $R$-matrix radius for which it is going to be used. Since two different radii are used in this work, two sets of continuum GTOs are necessary and we use those presented in Faure et al (2001). These GTOs are then orthogonalized to the ones being used for describing the target and only those with an overlap smaller than a certain threshold (in our case $2 \times 10^{-7}$ ) are retained.

\section{Results}

Since all the previous calculations concerning electron impact dissociative excitation of water used the fixed-nuclei approximation, we will first analyse our fixed-nuclei cross sections as well as the resonances for the equilibrium geometry.

The $1^{3} \mathrm{~A}^{\prime \prime}, 1^{1} \mathrm{~A}^{\prime \prime}$ and $1^{3} \mathrm{~A}^{\prime}$ states dissociate into $\mathrm{OH}\left(\mathrm{X}^{2} \Pi\right)+\mathrm{H}(1 \mathrm{~s})$, that is both fragments in their ground state. Conversely, the state $2^{1} \mathrm{~A}^{\prime}$ dissociates into the lowest excited state of the hydroxyl, $\mathrm{OH}\left(\mathrm{A}^{2} \Sigma^{+}\right)$, and the ground state of $\mathrm{H}$. In this last case, the equilibrium bond length of $\mathrm{OH}, 1.91$, differs more significantly from that of water, making our approximation of fixing this distance at 1.81 less satisfactory. Nevertheless, we think that the adiabatic nuclei cross section will give some idea of the effect of the nuclear motion on this process. We have tested the inclusion of the Born correction (Chu and Dalgarno 1974): this correction is non-zero only for dipole allowed transitions and therefore will only affect the cross sections for excitation into the $2^{1} \mathrm{~A}^{\prime}$ and $1^{1} \mathrm{~A}^{\prime \prime}$ states. Its maximum effect occurs for the latter at $15 \mathrm{eV}$ where the change in the cross section is smaller than $10 \%$. Consequently, we do not include this correction in the results shown in this section.

Resonances positions and widths are determined using two methods: by fitting the eigenphase sum to a Breit-Wigner profile (Tennyson and Noble 1984) and by fitting the largest eigenvalue of the time-delay matrix using the program TIMEDEL (Stibbe and Tennyson 1998b). 


\subsection{Fixed-nuclei cross sections and resonances}

We investigate the existence of a bound $\mathrm{H}_{2} \mathrm{O}^{-}$state. No bound states are found for $\mathrm{H}_{2} \mathrm{O}^{-}$ either using the CASCI model or using CASSD or CASSDT (CAS plus single, double and triple excitations) models. Morgan (1998) found a weakly bound state of symmetry ${ }^{2} \mathrm{~A}_{1}$ that is likely to become unbound if rotational motion is taken into account. We therefore conclude that there are no bound states for this system.

Since water is a polar molecule, the elastic cross section is formally divergent in the fixed-nuclei approximation. But it is possible to overcome this using a closure formula that involves the Born approximation (see, for example, Gianturco et al 1998) to calculate cross sections that depend on the initial rotational state.

The resulting cross section for $\mathrm{J}=0$ is plotted in figure 2. Agreement with similar results from Gianturco et al (1998) and Okamoto et al (1993) is very good. Experimental results (Johnstone and Newell 1991, Danjo and Nishimura 1985, Shyn and Cho 1987) are smaller over the whole energy range. As mentioned by Okamoto et al (1993) this is probably due to incorrect extrapolation of the differential cross sections to the forward direction needed since experiments were unable to properly measure the cross section both for small and large angles. The theoretical results of Varella et al (1999) agree better with experiment but they neglected target polarization.

We find a resonance of ${ }^{2} \mathrm{~B}_{1}\left({ }^{2} \mathrm{~A}^{\prime \prime}\right.$ in $\left.\mathrm{C}_{s}\right)$ symmetry below the first energy threshold that can be seen as a small peak in the elastic cross sections around $7 \mathrm{eV}$. Its position and width are shown in table 2 together with results from previous calculations and experiments. It is clear from our calculations that, in agreement with Belic et al, this is a Feshbach resonance whose main configuration and parent state are $1 b_{2}^{-1} 4 a_{1}^{2}$ and $1^{3} \mathrm{~B}_{1}$ (see figure $4 \mathrm{a}$ ) respectively. Morgan finds it at lower energies, in better agreement experiment; Gil et al find it above the first excitation threshold, but they argue that adjusting this threshold would bring it down around $1 \mathrm{eV}$. Since our threshold is already smaller than the recommended value of Winter et al (1975), it is highly unlikely that adjusting it would bring the resonance position down.

The cross sections for excitation into the four lowest electronic states all have similar magnitude. The most noticeable difference between them is the presence of a sharp threshold for the (dipole forbidden) transitions into the triplet states. The cross section for excitation into the state $1^{3} \mathrm{~B}_{1}$ is shown in figure 3 . The results for models (a) and (b) are very similar and they agree better with those of Gil et al (1994) than with Morgan's which show a wide peak at about $10 \mathrm{eV}$. This is somewhat surprising since Gil et al 's calculation is simpler and fairly different from ours, whereas Morgan used the same methodology and a similar model to describe the target and target plus electron wave functions. The main difference between Morgan calculation and ours is the basis set used to obtain the target molecular orbitals: Morgan used exactly the same one as Gil et al. We performed some tests using that basis set and concluded that the the peak at $10 \mathrm{eV}$ in Morgan's results is an artifact of calculation due to the amplitudes of some target wavefunctions being non-zero outside the $R$-matrix box. We therefore removed 
the most diffuse functions from the basis set and used a smaller one.

Our cross section shows a resonance at $9.15 \mathrm{eV}$ for both models. This is a ${ }^{2} \mathrm{~A}^{\prime}$ Feshbach resonance $\left({ }^{2} \mathrm{~A}_{1}\right.$ in $\mathrm{C}_{2 v}$ symmetry) that is observed at $8.6 \mathrm{eV}$ by Belic et al (1981) who attribute it the same main configuration, $3 \mathrm{a}_{1}^{-1} 4 \mathrm{a}_{1}^{2}$. Morgan finds it at lower energies, in better agreement with the experimental value (see table 2) but identifies it as a core excited shape resonance. Gil et al see it at higher energies, and again they argue that adjusting for the error in their threshold energy brings it down around $1 \mathrm{eV}$ to $9.3 \mathrm{eV}$. Once more our threshold is slightly lower than the recommended one. Our cross sections shows some structure in the $12-13 \mathrm{eV}$ region. This is probably due to the presence of $\mathrm{a}^{2} \mathrm{~A}^{\prime}\left({ }^{2} \mathrm{~B}_{2}\right.$ in $\left.\mathrm{C}_{2 v}\right)$ resonance that is a clearly visible feature in the cross sections for excitation into the ${ }^{3,1} \mathrm{~A}^{\prime}$ states. The very small bump around $12.3 \mathrm{eV}$ in the 9-state calculation is probably related to the $1^{3} \mathrm{~A}_{2}$ state, whose threshold energy is $12.338 \mathrm{eV}$.

In the case of the cross section for excitation into the ${ }^{1} \mathrm{~B}_{1}(\tilde{A})$ state, there are some differences between the 7 and 9 states calculation: their shape is different in the 8$13 \mathrm{eV}$ region. For higher energies both cross sections show similar behaviour but their magnitude differs by about $20 \%$. The resonances (seen as small peaks) appear at the same energies for both calculations: $12.97 \mathrm{eV}$ for the abovementioned ${ }^{2} \mathrm{~B}_{2}$ resonance and $9.15 \mathrm{eV}$ for the ${ }^{2} \mathrm{~A}_{1}$. Our cross sections are significantly bigger than those of Morgan and Gil et al in the whole energy range. Shifting the threshold to its recommended value of $7.49 \mathrm{eV}$ slightly increased Morgan's cross section: it still remained smaller than ours.

For excitation into the $1^{3} \mathrm{~A}_{1}$ state, once again the 7 and 9 states calculations give similar results (see figure 3). This is the cross section for which the largest number of results is available. Although all of them have similar magnitude to ours, there is little agreement on its shape. Both Pritchard et al (using the Schwinger method) and Lee et al (distorted wave calculation) performed two state calculations. The discrepancies between their results show that the differences between their and other cross sections is not only due to the number of states. The ${ }^{2} \mathrm{~A}^{\prime}\left({ }^{2} \mathrm{~B}_{2}\right)$ resonance at $12.97 \mathrm{eV}$ is clearly visible in this cross section. Morgan finds this resonance at slightly lower energies in better agreement with experimental results (see table 2). We believe this to be a Feshbach resonance, in accordance with Belic̀ et al who, like us, attribute it the $1 \mathrm{~b}_{2}^{-1} 4 \mathrm{a}_{1}^{2}$ configuration.

Finally, the cross section for excitation to the ${ }^{1} \mathrm{~A}_{1}(\tilde{B})$ state is shown in figure 3 . Our cross sections for both models are again very similar in shape, with a maximum $20 \%$ difference in the magnitude at $15 \mathrm{eV}$. Morgan's cross section is significantly bigger than ours: almost twice as big at $15 \mathrm{eV}$. Gil et al have performed two calculations generating the IVO orbital in two different ways. The calculation they feel is more reliable is in better quantitative agreement with that of Morgan for high energies, whereas the other agrees better with our results for the whole energy range. Once again, the ${ }^{2} \mathrm{~A}^{\prime}$ resonance is clearly visible at $12.97 \mathrm{eV}$.

Summing up the differences between 7 and 9 states calculation, the ${ }^{1} \mathrm{~B}_{1}(\tilde{A})$ is the 
only cross section that shows difference in its shape. The differences in the magnitudes of the other cross sections are never bigger than $20 \%$. Therefore, since the use of the 7 state basis set with $a=10$ is computationally cheaper, we have opted for it in the adiabatic-nuclei calculation.

In figure 4 we have plotted the position and width of the ${ }^{2} \mathrm{~B}_{1}$ and ${ }^{2} \mathrm{~A}_{1}$ resonances as a function of the $\mathrm{OH}$ bond length. Figure $4 \mathrm{~b}$ shows that the width of the ${ }^{2} \mathrm{~A}^{\prime \prime}\left({ }^{2} \mathrm{~B}_{1}\right)$ resonance has a maximum at the equilibrium $\mathrm{OH}$ bond length whereas the width of the ${ }^{2} \mathrm{~A}^{\prime}\left({ }^{2} \mathrm{~A}_{1}\right)$ resonance decreases with increasing $\mathrm{OH}$ distance. The position of the resonances follows very closely the energies of the $1^{3} \mathrm{~A}^{\prime \prime}$ and $1^{3} \mathrm{~A}^{\prime}$ states. For $\mathrm{r}_{O H}$ larger than 2.3 the two resonances become very narrow and we are unable to fit them. We were unable to fit the ${ }^{2} \mathrm{~B}_{2}$ resonance for all bond lengths because it is too close to the $1^{3} \mathrm{~B}_{1}$ threshold. Indeed, we believe that this resonance is probably not accurately described in our calculations since its parent state, the $1^{3} \mathrm{~B}_{2}$, is not well represented. Regardless of this, we have manually fitted the eigenphase to a Breit-Wigner profile and found approximate position and width for the equilibrium geometry.

\subsection{Adiabatic nuclei results}

To perform the adiabatic nuclei $(\mathrm{AN})$ calculation we assume that initially the $\mathrm{H}_{2} \mathrm{O}$ is in its ground vibrational state. We use the $T$-matrices for 14 different geometries obtained using model (a) ( 7 states and $a=10$ ) and changing $r_{O H}$ from 1.3 to 2.6 in steps of 0.1 . To test the convergence of the cross sections with the range of $\mathrm{OH}$ distances, we performed an initial 12 geometries calculation over the range [1.4;2.5]: differences between both sets of cross sections were negligible.

The most significant feature of the $\mathrm{AN}$ cross sections is the fact that they are different from zero below the equilibrium fixed-nuclei thresholds. This result is, of course, impossible to obtain in a fixed-nuclei calculation. For the singlets, the cross section starts to be sizeable less than $0.5 \mathrm{eV}$ below the fixed-nuclei (vertical) threshold. In the case of the triplets, around $1 \mathrm{eV}$ below it. The larger effect on the triplets is due to the sharp onset of the cross-sections for dipole-forbidden transitions. In principle, the dissociation cross section should be different from zero when the energy of the incoming electron is bigger than the dissociation energy $\mathrm{D}_{\nu}$ (in this case $5.2 \mathrm{eV}$ for $\nu=0$ ). But the threshold observed experimentally (called the effective Franck-Condon threshold) is different because it depends on the overlap between the initial and final vibrational wavefunctions. We find this effective threshold at $\approx 6 \mathrm{eV}$ (see figure 6) which appears consistent with the results of Harb et al (2001).

The adiabatic nuclei (AN) cross section for excitation into $1^{3} \mathrm{~A}^{\prime \prime}$, plotted in figure 5 , is similar to the fixed-nuclei one, but a bit smaller for all energies higher than the threshold energy. The adiabatic approximation does not treat resonances correctly so the resonance is averaged out in the nuclear motion calculations. The ${ }^{1} \mathrm{~A}^{\prime \prime}(\tilde{A}) \mathrm{AN}$ cross section is also similar to the fixed-nuclei one: the shape is very similar, with the AN cross section being slightly smaller for $\mathrm{E}>9 \mathrm{eV}$. 
In contrast, the $\mathrm{AN}$ cross sections for the $1^{3} \mathrm{~A}^{\prime}$ and ${ }^{1} \mathrm{~A}^{\prime}(\tilde{B})$ states differ more significantly from the fixed-nuclei nuclei ones. In the case of $1^{3} \mathrm{~A}^{\prime}$, the $\mathrm{AN}$ cross section is much bigger over the whole energy range (see figure 5) whereas the ${ }^{1} \mathrm{~A}^{\prime}(\tilde{B})$ one is smaller over the whole range. This last cross section also shows the smallest below vertical threshold values. The explanation for this can be found in the electronic energies of these states as a function of the internuclear separation and the effect this has on the nuclear continuum wave functions. For the ${ }^{1} \mathrm{~A}^{\prime}(\tilde{B})$ state, these functions have many more maxima and nodes in the $[1.3 ; 2.6]$ range than the corresponding functions for the other states. This will have the effect of cancelling the fixed-nuclei cross sections, thus giving a smaller AN one. The case of the $1^{3} \mathrm{~A}^{\prime}$ is exactly the opposite, with fewer maxima and nodes, making the AN cross section bigger than the fixed-nuclei one.

\subsection{Comparison with experiment. Limitations of our method.}

Figure 6 shows the total cross section for electron impact dissociative excitation measured by Harb et al (2001) who performed laser induced fluorescence measurements of $\mathrm{OH}\left(\mathrm{X}^{2} \Pi\right)$ production. According to them, the $1^{3} \mathrm{~A}_{1}$ and ${ }^{1} \mathrm{~A}_{1}(\tilde{B})$ states yield $\mathrm{OH}\left(\mathrm{A}^{2} \Sigma\right)$. For this reason we have plotted in figure 6 the sum of the AN cross sections for excitation into the $1^{3} \mathrm{~B}_{1}$ and ${ }^{1} \mathrm{~B}_{1}(\tilde{A})$ states as well as the sum of the cross sections for the four lowest states of water. Our results and those of Morgan (1998) are much bigger than the experimental results in the whole energy range. The total cross section of Gil et al (1994) seems to be in better agreement but this is due to their thresholds being to high.

The discrepancy between theory and experiment may be due to the limitations of our calculation: the overestimation of the cross section may be attributable to the use of a one-dimensional model to treat the nuclear motion. A full three-dimensional treatment would allow other processes, including both relaxation and other dissociation paths to take place, thus reducing the probability that flux goes into the $\mathrm{OH}+\mathrm{H}$ channel. Avoided crossing, which can not be treated in the adiabatic-nuclei approximation, could become relevant. For example, there is a well known conical intersection that plays an important role in photodissociation dynamics (see van Harrevelt and van Hemert 2000).

Furthermore, it is well known that the AN approximation fails in the proximity of resonances. The resonances found for water in the energy range treated in this paper are narrow and therefore long-lived. When this is the case, an accurate treatment of nuclear motion in the resonant state potential energy surface is required. However, our model assumes that the dominant contribution to the dissociation cross section is nonresonant; the lack of structure in the experimental results could be taken as to confirm this assumption. Finally, the use of the $R$-matrix method introduces a limitation in the diffuseness of the wavefunctions describing the target states. As a consequence the polarizability of the molecule may not be accurately reproduced and the quality of the representation of those states of Rydberg character is undermined. 


\section{Conclusions}

We have calculated the electron impact dissociative excitation cross sections for the four lowest excited states of water using the energy balancing approximation. The cross sections calculated with this method have non-negligible values below the fixed-nuclei vertical threshold and although the magnitude of the cross sections for the $1^{3} \mathrm{~A}^{\prime \prime}$ and $1^{1} \mathrm{~A}^{\prime \prime}$ states is very similar to the fixed-nuclei ones those of the $1^{3} \mathrm{~A}^{\prime}$ and $2^{1} \mathrm{~A}^{\prime}$ states differ significantly. The present calculations are the first to consider the nuclear motion in electron impact dissociation of a polyatomic molecule. Nevertheless, there is room for improvement: so far we have only attempted a one dimensional calculation. It remains to be seen if a full three-dimensional study that would allow, for example, asymmetric distortion, will significantly alter the results.

\section{Acknowledgments}

This work was supported by the UK Engineering and Physical Sciences Research Council. We are are thankful to J. W. McConkey for providing us with his results prior to publication.

\section{References}

Belic̀ D S, Landau M and Hall R I 1981 J. Phys. B: At. Mol. Opt. Phys. 14175

Boudaiffa B, Cloutier P, Hunting D, Huels M A and Sanche L 2000 Science 2875458

Chase D M 1956 Phys. Rev. 104838

Chu S I and Dalgarno A 1974 Phys. Rev. A 10788

Curtis M G and Walker I C 1992 J. Chem. Soc. Faraday Trans. 882805

Danjo A and Nishimura H 1985 J. Phys. Soc. Jap. 541224

do N Varella M T, Bettega M H F, Lima M A P and Ferreira L G 1999 J. Chem. Phys. 1116396

Dunning Jr. T H 1970 J. Chem. Phys. 532823

Dunning Jr. T H 1971 J. Chem. Phys. 55716

V Engel, V Staemmler, R L Vander Wal, F F Crim, R J Sension, B Hudson, P Andresen, S Hennig, K Weide, and R Schinke 1992 J. Phys. Chem. 963201

Faure A, Gorfinkiel J D, Morgan L A and Tennyson J 2001 Comput. Phys. Commun. in press.

Gianturco F A, Meloni S, Paioletti P, Lucchese R R and Sanna N 1998 J. Chem. Phys. 1084002

Gil T J, Rescigno T N McCurdy C W and Lengsfield B H 1994 Phys. Rev. A 492642

Hara S 1969 J. Phys. Soc. Jap. 271592

Harb T, Kedzierski W and McConkey J W 2001 J. Chem.Phys. 17123

Hwang D W, Yang X F, Harich S, Lin J J and Yang Y 1999 J. Chem.Phys. 1104123

Johnstone W M and Newell W R 1991 J. Phys. B: At. Mol. Opt. Phys. 243633

Kimmel G A and Orlando T M 1995 Phys. Rev. Letts. 752606

Kurawaki J, Ueki K, Higo M and Ogawa J 1983 J. Chem. Phys. 783071

Lassetre E N, Skerbele A, Dillon M A and Ross K J 1968 J. Chem.Phys.21 145

Lee M T, Michelin S E, Machado L E and Brescansin L M 1993 J. Phys. B: At. Mol. Opt. Phys. 26 L203

Le Roy R J 1996 University of Waterloo Chemical Physics Research Report CP-555R

Melton C E 1972 J. Chem. Phys. 574218 
Mordaunt D H, Ashfold M N R and Dixon R N 1994 J. Chem. Phys. 1007360

Morgan L A, Gillan C J, Tennyson J and Chen X 1997 J. Phys. B: At. Mol. Opt. Phys. 304087

Morgan L A, Tennyson J and Gillan C J 1998 Computer Phys. Commun. 114120

Morgan L A 1998 J. Phys. B: At. Mol. Opt. Phys. 315003

Nestmann B M and Peyerimhoff S D 1990 J. Phys. B: At. Mol. Opt. Phys. 23 L773

NIST, Standard Reference Database 69 - July 2001 Release: NIST Chemistry WebBook, webbook.nist.gov/chemistry/

Okamoto Y, Onda K and Itikawa Y 1993 J. Phys. B: At. Mol. Opt. Phys. 26745

Pritchard H P, McKoy V and Lima M A P 1990 Phys. Rev. A 41546

Rountree P, Parentenau L and Sanche L 1991 J. Chem. Phys. 948570

Sarpal B K, Pfingst K, Nestmann B M and Peyerimhoff S D 1996 J. Phys. B: At. Mol. Opt. Phys. 29 857

Schinke R 1993 Photodissociation Dynamics (Cambridge University Press)

Shugard M. and Hazi A. U. 1975 Phys. Rev. A, 12, 1895

Shyn T W and Cho S Y 1987 Phys. Rev. A 365138

Stibbe D. T and Tennyson J 1998 a New J. Phys. 1 2.1-2.9

Stibbe D T and Tennyson J 1998b Computer Phys. Comms. 114236

Tennyson J and Noble C J 1984 Comput. Phys. Commun. 32421

Tennyson J 1996 J. Phys. B: At. Mol. Opt. Phys. 296185

Trajmar S, Williams W and Kupperman A 1973 J. Chem. Phys. 582521

Trevisan C S and Tennyson J 2001 J. Phys. B: At. Mol. Opt. Phys. 342935

Tsurubichi S, Iwai T and Horie T 1974 J. Phys. Soc. Jap. 36537

van Harrevelt R and van Hemert M C 2000 J. Chem. Phys. 1125777

van Harrevelt R and van Hemert M C 2000 J. Chem. Phys. 1125787

Winter N W, Goddard IIII W A and Bobrowicz F W 1975 J. Chem. Phys. 624325

Yang X F, Hwang D W, Lin J J and Ying X 2000 J. Chem. Phys. 11310597 


\section{Tables and table captions}

Table 1. Vertical energy excitations (in eV) for the $\mathrm{H}_{2} \mathrm{O}$ target states. Also given are the number of configurations, $N$, in the $\mathrm{CI}$ and excitation values from: (A) van Harrevelt and van Hemert (2000) and (B) best values from Winter et al (1975).

\begin{tabular}{|c|c|c|c|c|c|}
\hline \multicolumn{2}{|c|}{ State } & \multirow[b]{2}{*}{$N$} & \multicolumn{2}{|c|}{ Vertical excitation } & \multirow{2}{*}{$\frac{(\mathrm{eV})}{\mathrm{B}}$} \\
\hline $\mathrm{C}_{2 v}$ & $\mathrm{C}_{s}$ & & This work & $\mathrm{A}$ & \\
\hline${ }^{1} \mathrm{~A}_{1}(\tilde{X})$ & ${ }^{1} \mathrm{~A}^{\prime}(\tilde{X})$ & 260 & - & - & - \\
\hline $1^{3} \mathrm{~B}_{1}$ & $1^{3} \mathrm{~A}^{\prime \prime}$ & 298 & 7.034 & - & 7.14 \\
\hline${ }^{1} \mathrm{~B}_{1}(\tilde{A})$ & ${ }^{1} \mathrm{~A}^{\prime \prime}(\tilde{A})$ & 230 & 7.508 & 7.63 & 7.49 \\
\hline $1^{3} \mathrm{~A}_{1}$ & $1^{3} \mathrm{~A}^{\prime}$ & 290 & 9.202 & - & 9.35 \\
\hline${ }^{1} \mathrm{~A}_{1}(\tilde{B})$ & ${ }^{1} \mathrm{~A}^{\prime}(\tilde{B})$ & 260 & 9.855 & 9.95 & 9.73 \\
\hline $1^{3} \mathrm{~A}_{2}$ & $2^{3} \mathrm{~A}^{\prime \prime}$ & 298 & 12.338 & - & \\
\hline $1^{3} \mathrm{~B}_{2}$ & $2^{3} \mathrm{~A}^{\prime}$ & 290 & 12.973 & - & 9.93 \\
\hline${ }^{1} \mathrm{~A}_{2}$ & ${ }^{1} \mathrm{~A}^{\prime \prime}$ & 230 & 13.123 & 9.60 & \\
\hline${ }^{1} \mathrm{~B}_{2}\left(\tilde{E}^{\prime}\right)$ & ${ }^{1} \mathrm{~A}^{\prime}\left(\tilde{E}^{\prime}\right)$ & 260 & 14.023 & 11.11 & 10.0 \\
\hline
\end{tabular}

Table 2. Symmetry, parent state, main configuration, resonance position, $\mathrm{E}_{r}$, and resonance width, $\Gamma_{r}$, in eV: this work, Belic et al (1981) (A), Morgan (1998) (B) and Gil et al (1994) (C).

\begin{tabular}{ccc|cc|c|cc|cc}
\hline \multirow{2}{*}{ Sym. } & \multirow{2}{*}{$\begin{array}{c}\text { Main } \\
\text { configuration }\end{array}$} & Parent & \multicolumn{2}{|c|}{ This work } & $\mathrm{A}$ & \multicolumn{2}{|c|}{$\mathrm{B}$} & \multicolumn{2}{c}{$\mathrm{C}$} \\
\cline { 4 - 10 } & state & $\mathrm{E}_{r}^{a}$ & $\Gamma_{r}^{a}$ & $\mathrm{E}_{r}$ & $\mathrm{E}_{r}$ & $\Gamma_{r}$ & $\mathrm{E}_{r}$ & $\Gamma_{r}$ \\
\hline${ }^{2} \mathrm{~B}_{1}$ & $1 \mathrm{~b}_{1}^{-1} 4 \mathrm{a}_{1}^{2}$ & $1^{3} \mathrm{~B}_{1}$ & 6.994 & 0.004 & 6.5 & 6.785 & 0.01 & 8.2 & - \\
${ }^{2} \mathrm{~A}_{1}$ & $3 \mathrm{a}_{1}^{-1} 4 \mathrm{a}_{1}^{2}$ & $1^{3} \mathrm{~A}_{1}$ & 9.151 & 0.010 & 8.6 & 8.81 & 0.02 & 10.5 & $\approx 0.2$ \\
${ }^{2} \mathrm{~B}_{2}$ & $1 \mathrm{~b}_{2}^{-1} 4 \mathrm{a}_{1}^{2}$ & $1^{3} \mathrm{~B}_{2}$ & $\approx 12.97$ & $\approx 0.1$ & 11.8 & 12.70 & 0.08 & - & - \\
\hline
\end{tabular}




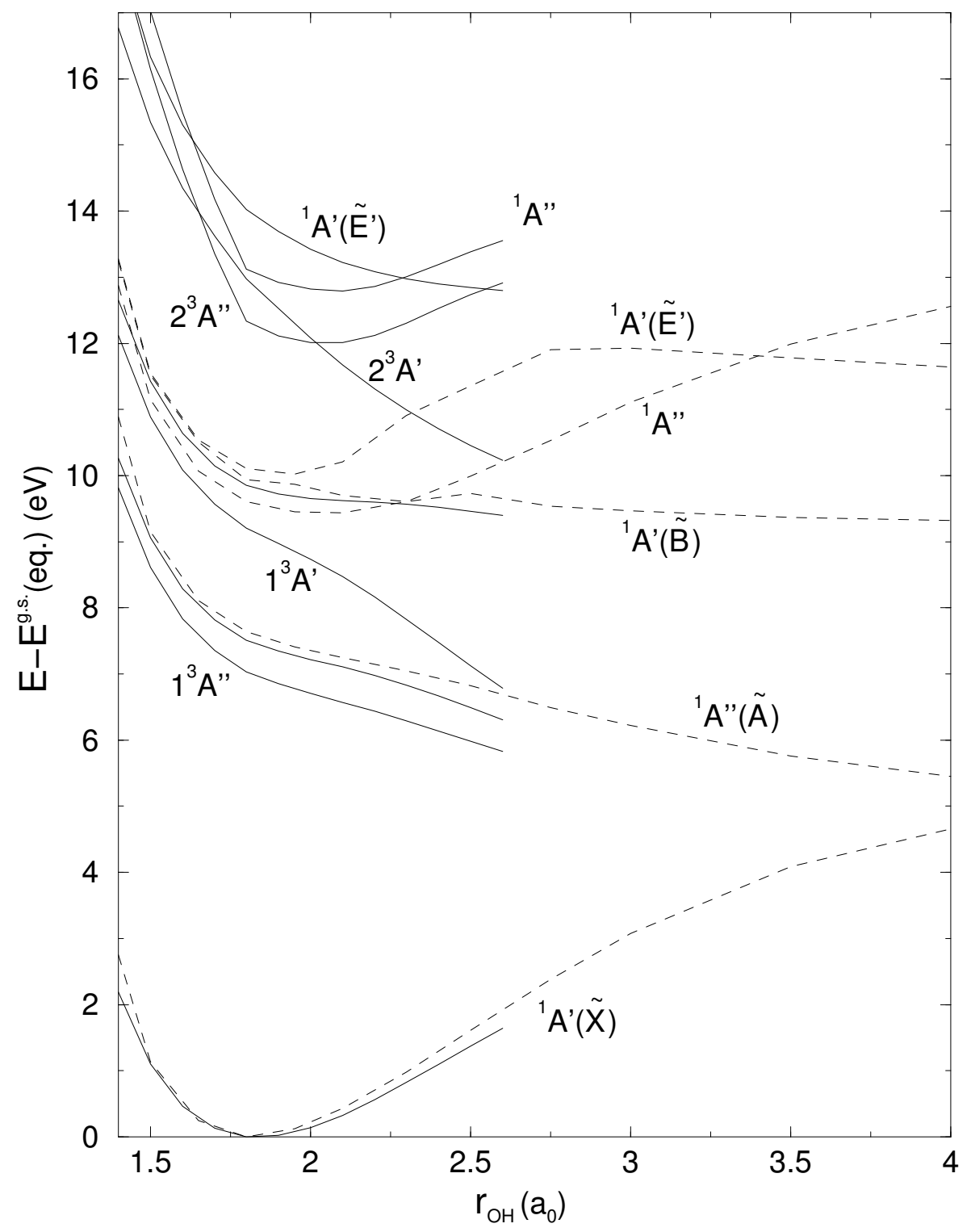

Figure 1. Energies of the 9 lowest electronic states of the $\mathrm{H}_{2} \mathrm{O}$ as a function of the bond length $r_{O H}$. The other bond length and the angle between both are kept at their equilibrium values. Full lines, this work; dashed lines, data for singlet states from (van Harrevelt and van Hemert 2000). Energies defined by setting the ground state equilibrium value of both calculations to zero. 


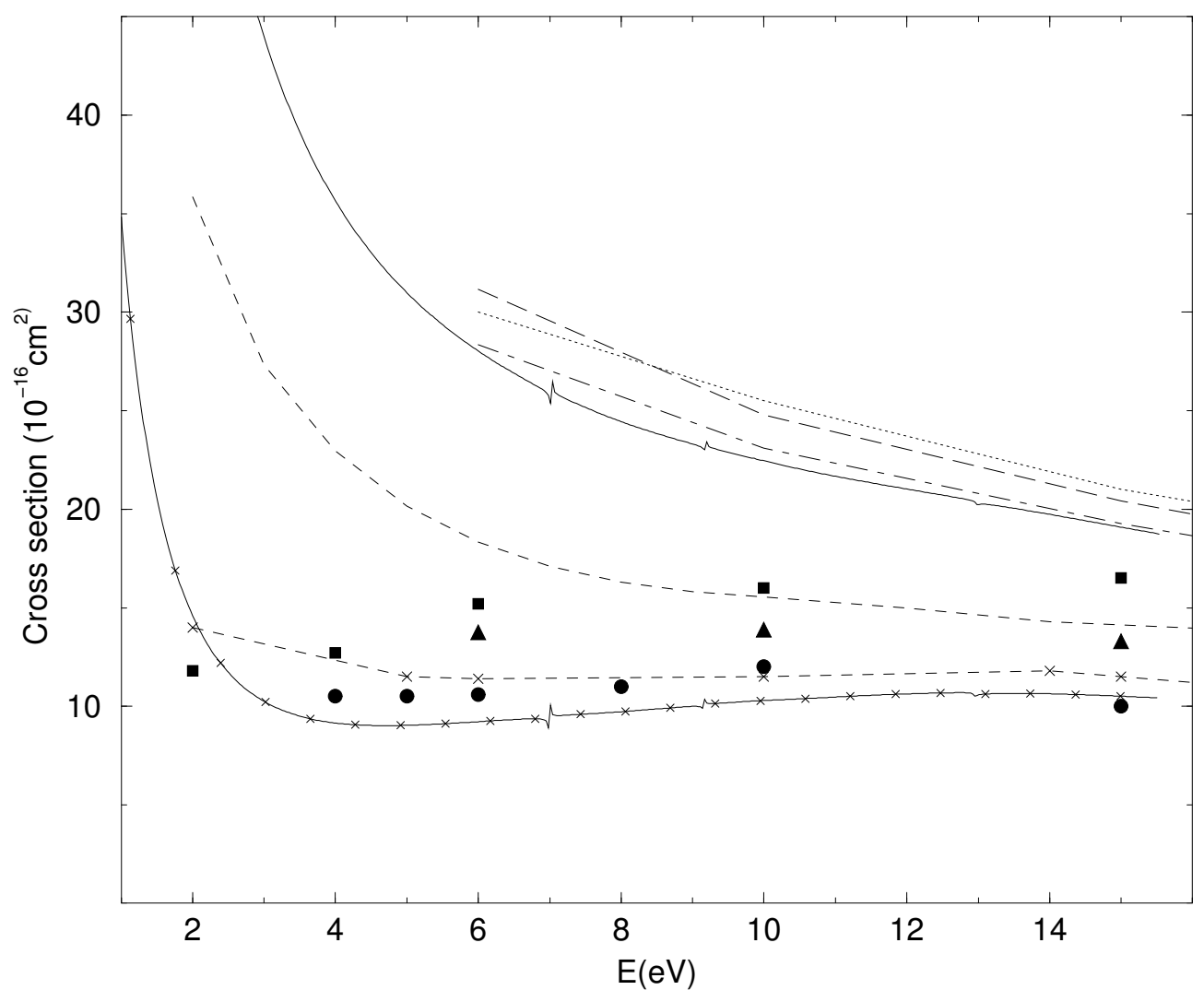

Figure 2. Electronically elastic cross section. Full line: Born corrected cross-section for $\mathrm{J}=0$; full line with $\mathrm{x}$ : $R$-matrix elastic cross section with $l \leq 4$ and model (a); dotted line: Gianturco et al (1998); long-dashed line: Okamoto et al (1993); dotted-dashed line: Okamoto et al (1993) averaged over rotational-state population; dashed-line: Varella et al (1999) with Born closure; dashed-line with x: same work without Born closure. Experimental results: triangles: Danjo and Nishimura (1985); circles Shyn and Cho (1987); full squares: Johnstone and Newell (1991). 

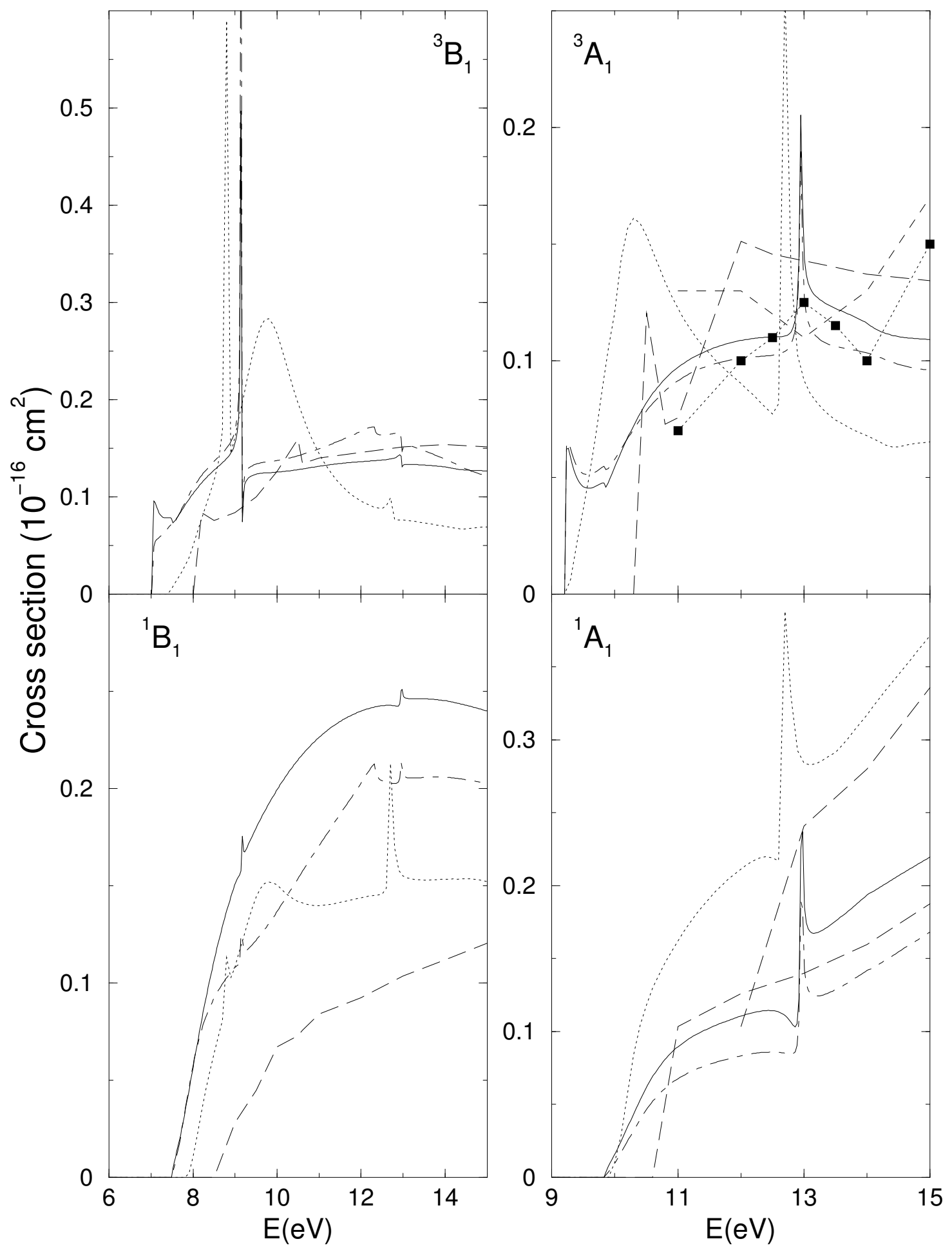

Figure 3. Fixed-nuclei cross sections for excitation into the four lowest excited states of $\mathrm{H}_{2} \mathrm{O}$ indicated in the figures. Full lines: this work, model (a); dotted-dashed line: this work, model (b); dotted line: Morgan (1998); long-dashed line: Gil et al (1994); dashed-line: Pritchard et al (1990) and dotted-line with squares: Lee et al (1993). 


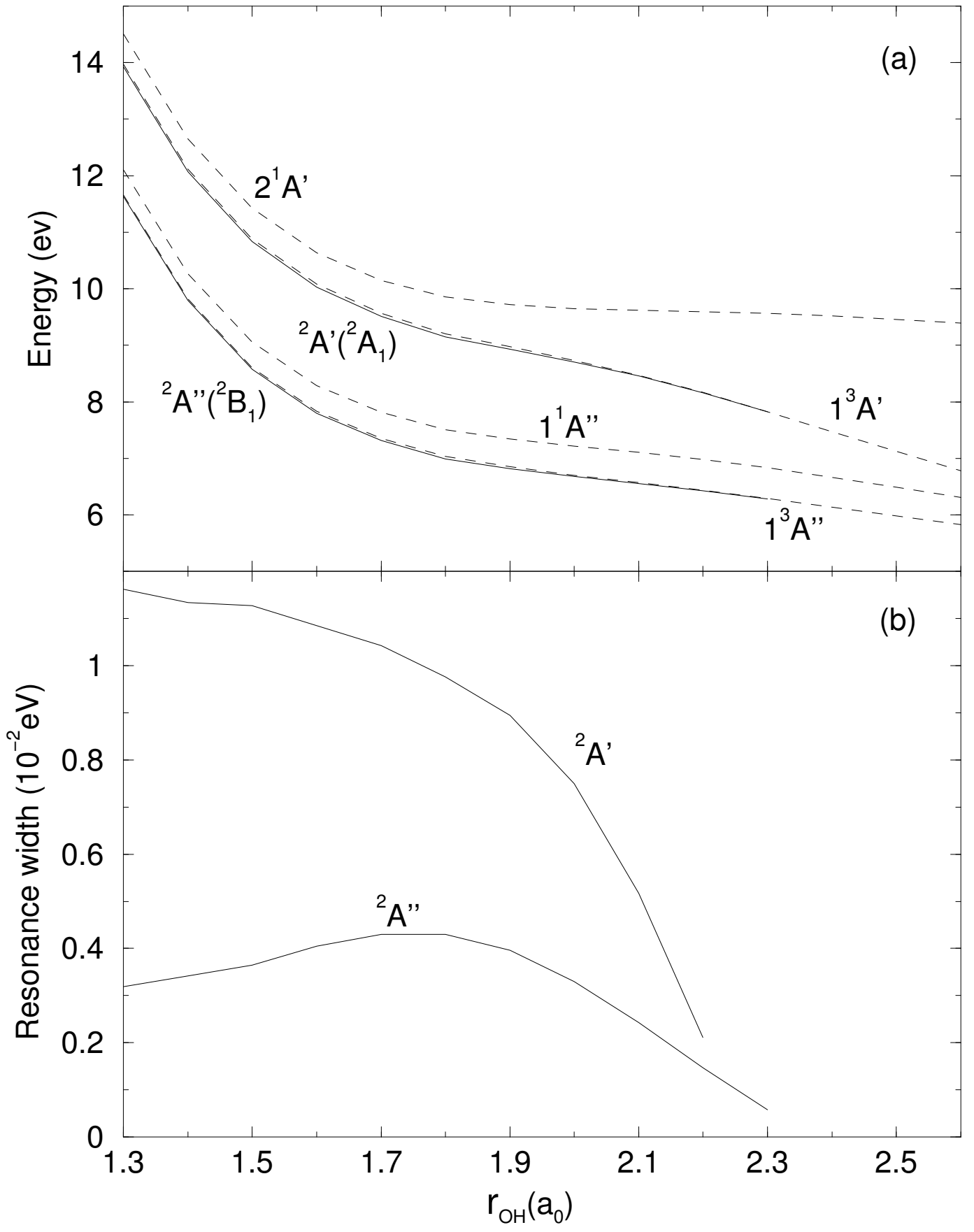

Figure 4. Resonance positions (a) and widths (b) as a function of the $\mathrm{OH}$ bond length for the resonances indicated in the figure. (a): Dashed-lines: threshold energies of the 4 lowest excited states; full lines: resonance positions as differences from the ground state equilibrium energy. 

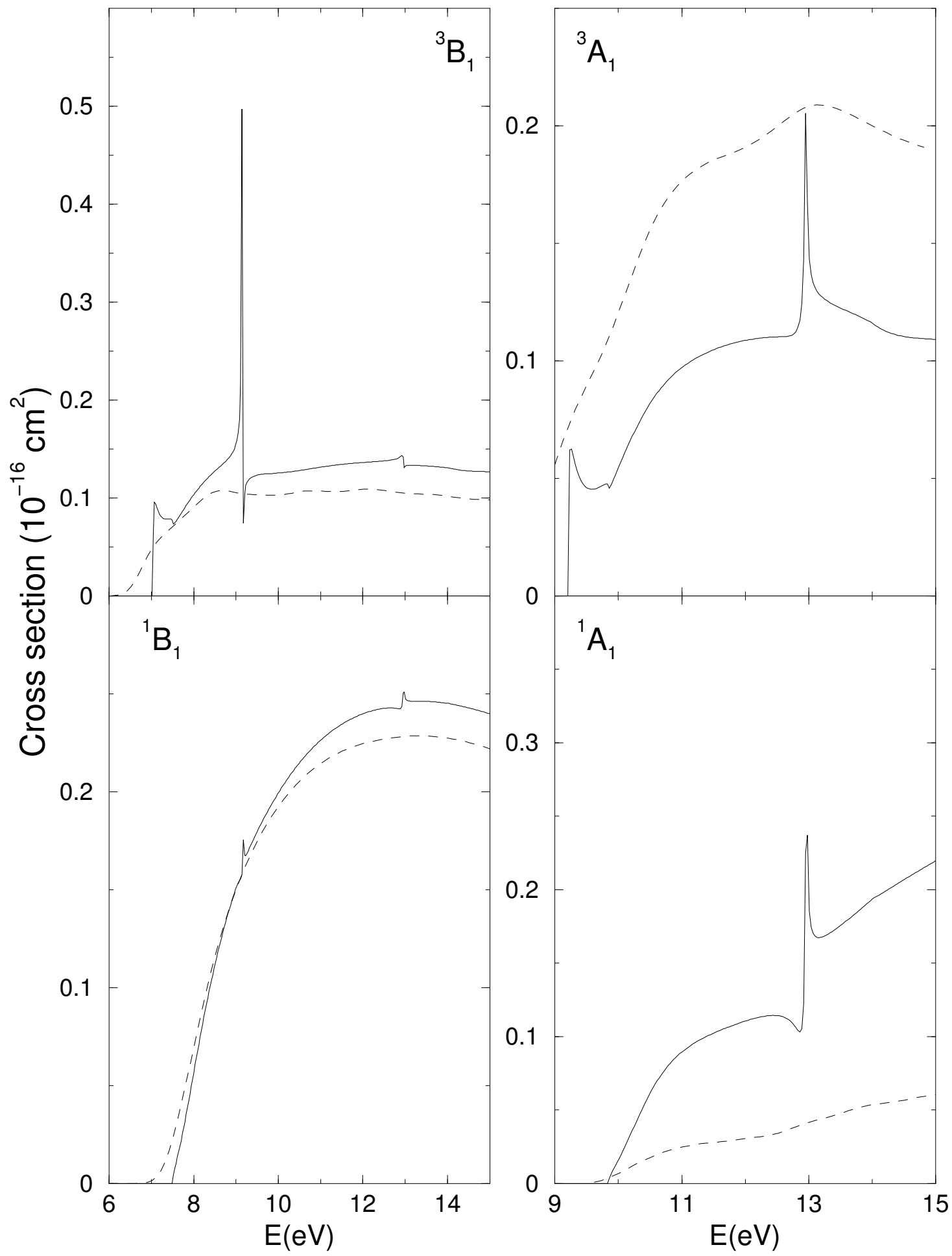

Figure 5. Total cross sections for excitation into the 4 lowest excited states of $\mathrm{H}_{2} \mathrm{O}$ indicated in the figures. Full lines: fixed-nuclei calculation; dashed-line: adiabatic nuclei calculation. Both calculations performed using model (a). 


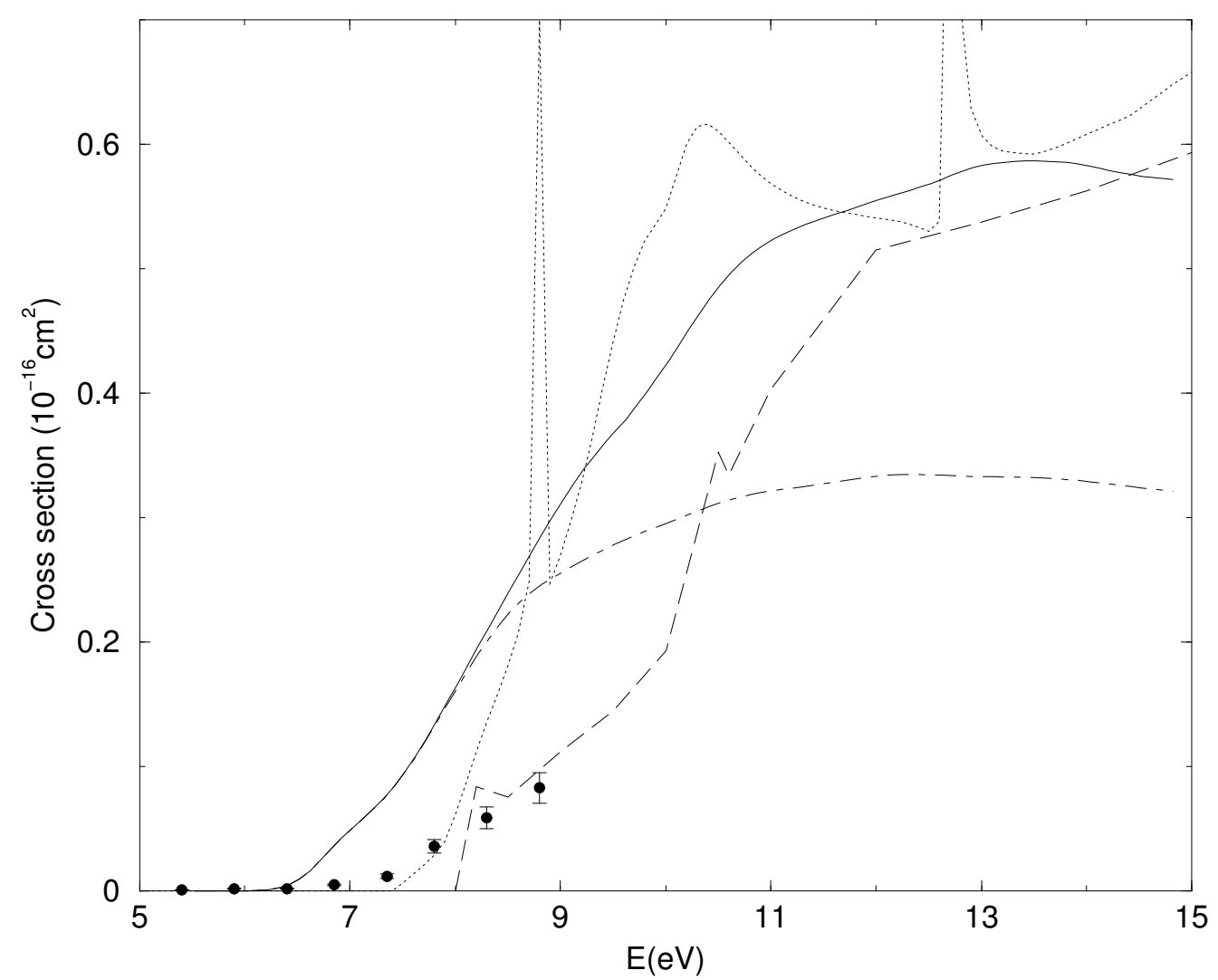

Figure 6. Comparison with experimental cross sections. Circles: direct dissociation measurements of Harb et al (2001). Full line: sum of the 4 calculated excitation AN cross sections. Dotted-dashed line: sum of the ${ }^{3} \mathrm{~B}_{1}$ and ${ }^{1} \mathrm{~B}_{1}(\tilde{A})$ AN cross sections. Dotted line: sum of Morgan's (1998) cross sections. Dotted-dashed lines:sum of Gil et al 's (1994) cross sections. 